Musées, Patrimoine et Culture scientifiques et techniques

$115 \mid 2008$

janvier - février 2008

\title{
Le Jardin des Explorateurs
}

Un nouveau projet pour les familles au Jardin botanique et à

l'Insectarium de Montréal

Johanne Landry

\section{(Q) OpenEdition \\ Journals}

Édition électronique

URL : http://journals.openedition.org/ocim/272

DOI : $10.4000 /$ ocim. 272

ISSN : 2108-646X

Éditeur

OCIM

Édition imprimée

Date de publication : 1 février 2008

Pagination : 6-7

ISSN : 0994-1908

Référence électronique

Johanne Landry, « Le Jardin des Explorateurs », La Lettre de I'OC/M [En ligne], 115 | 2008, mis en ligne

le 10 novembre 2010, consulté le 30 avril 2019. URL : http://journals.openedition.org/ocim/272 ; DOI :

$10.4000 /$ ocim. 272

Ce document a été généré automatiquement le 30 avril 2019.

Tous droits réservés 


\title{
Le Jardin des Explorateurs
}

\author{
Un nouveau projet pour les familles au Jardin botanique et à \\ l'Insectarium de Montréal
}

Johanne Landry

1 Le Jardin botanique et l'Insectarium de Montréal font partie, avec le Biodôme et le Planétarium de Montréal, des Muséums nature de Montréal. Le Jardin botanique de Montréal a été réalisé en 1931 et accueille annuellement 900000 visiteurs, dont 65000 enfants en visite scolaire. L'Insectarium de Montréal est établi sur le site du Jardin botanique depuis 1990 et accueille à lui seul 350000 visiteurs par année. Les Muséums nature ont pour mission de faire connaître la nature et les savoirs qui s'y rattachent, de contribuer à l'étude et à la préservation de la biodiversité et de promouvoir des comportements responsables face à l'environnement.

Depuis 2001, les Muséums nature de Montréal ont amorcé une planification afin d'identifier leurs stratégies de développement et leurs priorités pour les années 2002 à 2006. Dans le cadre de cette planification, une réflexion, tenue à l'automne 2005, a permis de dégager le potentiel de développement pour la clientèle familiale et les enfants sur le site du Jardin botanique et de l'Insectarium de Montréal. L'identification de pôles d'attraction pour la famille et les enfants s'est notamment appuyée sur une vaste enquête menée auprès de la clientèle des Muséums nature : Étude sur les besoins, les attentes et la satisfaction de la clientèle - Profil global des Muséums nature de Montréal. Cette étude révèle, si l'on combine la basse et la haute saison, que $37 \%$ des visiteurs du Jardin botanique sont accompagnés d'enfants, ce pourcentage s'élevant à $49 \%$ pour l'Insectarium de Montréal.

Confirmant ainsi sa clientèle majoritairement familiale et étant identifié par d'autres sondages comme l'un des musées favoris des enfants, l'Insectarium de Montréal a élaboré en 2006 un Plan directeur Diffusion et mise en valeur 2006-2011 qui vient synthétiser l'ensemble des priorités et des actions pour les cinq prochaines années. Le pourtour de l'Insectarium représente une zone sous-exploitée qui mérite une intervention à court et moyen terme. Elle comporte actuellement une aire de jeux pour enfants qui est constamment utilisée bien que fort désuète. Cette zone compte également plusieurs avantages qui justifient d'y développer une offre enfants/familles plus complète et plus 
attrayante : un stationnement à proximité, un positionnement stratégique par rapport aux autres pôles d'attraction du Jardin botanique (les jardins culturels) et une aire de restauration. Il est également important de souligner que la clientèle majoritaire du Jardin botanique y vient (en haute saison, à $60 \%$ ) pour se relaxer, passer une belle journée à l'extérieur et se divertir. Le développement de ce pôle d'activités au pourtour de l'Insectarium se fait donc en complémentarité et dans le respect des attentes de cette clientèle acquise et fidèle, qui ne recherche pas la même expérience de visite.

C'est donc à partir de ces constatations que les Muséums nature de Montréal ont entrepris de développer le projet du Jardin des Explorateurs, afin de créer un pôle d'attraction majeur et d'améliorer l'offre éducative et les services pour la clientèle familiale et les enfants. Ce projet développé par la firme d'architecture de paysage et de muséographie Schème au cours de l'année 2006-2007, est constitué de quatre aires extérieures adjacentes qui formeront un tout nouveau secteur du Jardin botanique de Montréal.

\section{Le Labyrinthe des découvertes}

5 Un tout nouveau labyrinthe en forme spectaculaire de feuille lancéolée sera dédié au thème de la biodiversité végétale. Les installations éducatives qu'on y trouvera visent à intéresser plus particulièrement les enfants de 8 à 12 ans. La forme des chambres du labyrinthe est inspirée des cellules qui composent une feuille et celle des sentiers rappelle ses nervures. Le labyrinthe est doté d'un belvédère d'observation, il est divisé en quatre parties thématiques liées aux plantes: "Nourrir» (importance des plantes en tant que principale source alimentaire), "Abriter» (utilité des plantes pour abriter humains et animaux), «Évolution des plantes terrestres », «Protéger la biodiversité ». Chacune des quatre chambres principales proposera des activités et des jeux de découverte du monde végétal et de la biodiversité.

\section{Sur la piste des insectes}

6 Ce tout nouveau Jardin des insectes du Québec et son kiosque d'animation extérieure (L'Agora), comportera une volière à papillons, des activités éducatives, un parcours de découvertes à travers trois habitats différents : un pré fleuri, un étang et un bois. Il sera divisé en six sections, chacune associée à un ordre d'insectes du Québec. Deux parcours d'exploration y seront proposés, chacun bordé de différents éléments d'interprétation: des insectes naturalisés présentés sous globe d'acrylique clair; une petite volière à papillons, incluant un jeu d'association des stades de métamorphose des papillons; un étang équipé d'une lunette d'observation d'insectes aquatiques; une agora pour les animations; cinq jeux interactifs d'imitation d'insectes (grillon, sauterelle, abeille, libellule, coléoptère).

\section{Terre de jeux}

7 Cette aire de jeux inédite sur le thème des nids d'insectes sera composée de deux sections qui répondent aux besoins de deux groupes d'âge d'enfants : les 18 mois à 5 ans et les 6 à 
12 ans. Elle comprendra cinq types de jeux : "Dômes d'insectes ", « Alvéoles d'une ruche en 3D », « Coupoles de larves d'abeilles », « Nid bulle d'air », « Toiles d'araignées ».

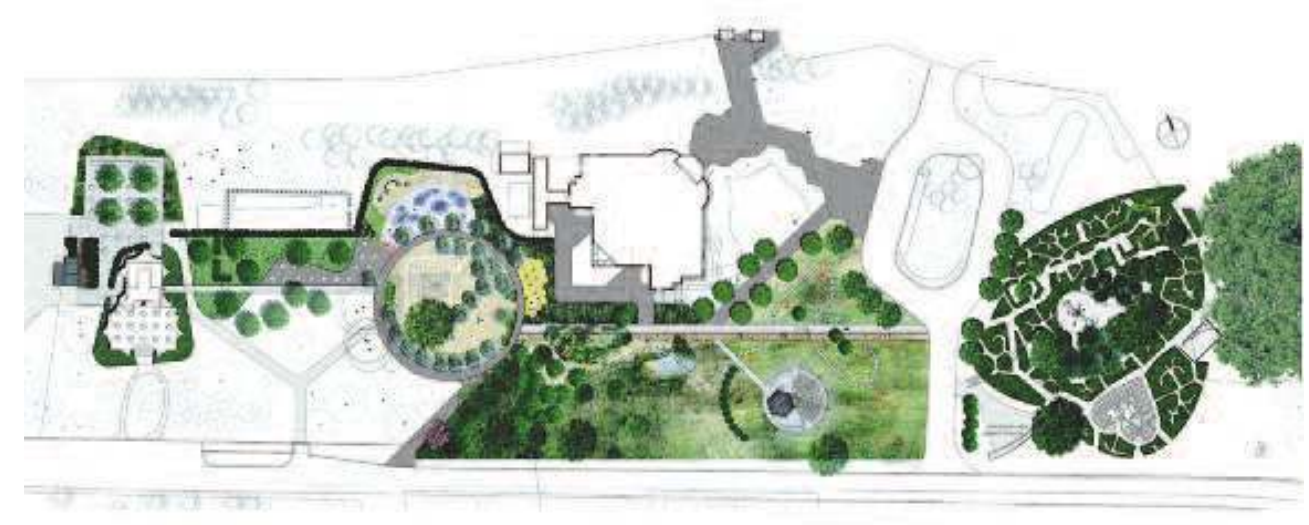

Positionnement des aires du projet sur le site du Jardin botanique avec de gauche à droite : l'aire de pique-nique, l'aire de jeux, le Jardin des insectes du Québec, le Labyrinthe végétal.

(c) Muséums nature de Montréal

\section{Le Resto-terrasse L'Amiral}

On créera une nouvelle aire de pique-nique pour les familles et deux terrasses seront réaménagées autour du restaurant du Pavillon Fuji, près de l'Insectarium. Initialement, ce restaurant extérieur pouvait accueillir 80 convives. Une fois réaménagé, le nombre de places assises passera à 240. De plus, afin de mieux lier cette aire à l'ensemble des aires du Jardin des Explorateurs, des possibilités d'apprentissage ludique sur le thème des fleurs insectivores et des pièces buccales d'insectes seront offertes aux visiteurs sous la forme d'intrigants panneaux à bascule proposant chacun une devinette.

Conjointement coordonné par la direction de l'Insectarium de Montréal et celle du Jardin botanique de Montréal, porté par l'ensemble du personnel des deux institutions et notamment Francine Hoffman (Jardin botanique), Elaine Boileau, (Insectarium) et Louise Lalonde (Jardin botanique et Insectarium), le projet du Jardin des explorateurs est en cours de développement. Les Muséums nature de Montréal collaborent depuis décembre 2006 avec la firme Schème pour élaborer le concept et le design des quatre aires pour enfants et familles. Cette étape est terminée et nous nous apprêtons à lancer les appels d'offres pour la réalisation finale du projet qui devrait intervenir au printemps 2009. 
INDEX

Mots-clés : enfants, famille, Insectarium, jeux

Index géographique : Canada, Montréal

AUTEUR

JOHANNE LANDRY

Directrice de l'Insectarium de Montréal, Muséums nature de Montréal 